\title{
A Strict Correlation between Dendritic and Somatic Plateau Depolarizations in the Rat Prefrontal Cortex Pyramidal Neurons
}

\author{
Bogdan A. Milojkovic, ${ }^{1,2}$ Mihailo S. Radojicic, ${ }^{1}$ and Srdjan D. Antic ${ }^{1,3}$ \\ ${ }^{1}$ Department of Neurobiology, Yale University, New Haven, Connecticut 06520-8001, ${ }^{2}$ Department of Neuroscience, Erasmus Medical Center, 3015 GE \\ Rotterdam, The Netherlands, and ${ }^{3}$ Department of Neuroscience, University of Connecticut Health Center, Farmington, Connecticut 06030
}

\begin{abstract}
One of the fundamental problems in neurobiology is to understand the cellular mechanism for sustained neuronal activity (neuronal UP states). Prefrontal pyramidal neurons readily switch to a long-lasting depolarized state after suprathreshold stimulation of basal dendrites. Analysis of the dendritic input- output function revealed that basal dendrites operate in a somewhat binary regimen (DOWN or UP) in regard to the amplitude of the glutamate-evoked electrical signal. Although the amplitude of the dendritic potential quickly becomes saturated (dendritic UP state), basal dendrites preserve their ability to code additional increase in glutamatergic input. Namely, after the saturation of the plateau amplitude, an additional increase in excitatory input is interpreted as an increase in plateau duration. Experiments performed in tetrodotoxin indicate that the maintenance of a stable depolarized state does not require inhibitory inputs to "balance" the excitation. In the absence of action potential-dependent (network-driven) GABAergic transmission, pyramidal neurons respond to brief $(5 \mathrm{~ms})$ glutamate pulses with stable long-lasting $(\sim 500 \mathrm{~ms})$ depolarizations. Voltage-sensitive dye recordings revealed that this somatic plateau depolarization is precisely time-locked with the regenerative dendritic plateau potential. The somatic plateau rises a few milliseconds after the onset of the dendritic transient and collapses with the breakdown of the dendritic plateau depolarization. In our in vitro model, the stable long-lasting somatic depolarization (UP state like) is a direct consequence of the local processing of a strong excitatory glutamatergic input arriving on the basal dendrite. The slow component of the somatic depolarization accurately mirrors the glutamate-evoked dendritic plateau potential (dendritic UP state).
\end{abstract}

Key words: basal dendrites; glutamate; voltage-sensitive dyes; dendritic recordings; plateau potentials; UP states

\section{Introduction}

Pyramidal neurons in vivo exhibit characteristic depolarizing shifts in membrane potential known as UP states, and it is only during UP states that these neurons fire action potentials (APs) (Steriade et al., 1993a; Lewis and O'Donnell, 2000). The somatic depolarization is stable throughout the entire duration of the UP phase (several hundred milliseconds), regardless of the frequency of APs (Steriade et al., 1993b), or even in the absence of APs (Metherate and Ashe, 1993; Steriade et al., 1993a). So far, it has not been possible to obtain an adequate explanation for the existence of an equilibrium state associated with the depolarized state. For example, if a pyramidal neuron in vivo is depolarized by somatic current injection, the cell body will not show any tendency to maintain the depolarized state when the current is turned off (West et al., 2002). Likewise, a hyperpolarizing current will not terminate a spontaneous depolarizing episode (Steriade et al., 1993a; Cowan and Wilson, 1994).

If intrinsic somatic membrane properties are not accountable

Received June 1, 2004; revised Feb. 20, 2005; accepted March 12, 2005.

This work was supported by National Institutes of Health Grant MH063503.

Correspondence should be addressed to Dr. Srdjan D. Antic, Department of Neuroscience, L-4000, University of Connecticut Health Center, 263 Farmington Avenue, Farmington, CT 06030-3401. E-mail: antic@neuron.uchc.edu. D01:10.1523/JNEUROSCI.5314-04.2005

Copyright $\odot 2005$ Society for Neuroscience $\quad$ 0270-6474/05/253940-12\$15.00/0 for the maintenance of the UP state, then what is the mechanism responsible for sustained plateau depolarization? The prevalent hypothesis says that the stable period of activity (UP state) is based on the fine balance between recurrent excitation and inhibition. According to this hypothesis, the generation of persistent cortical activity is associated with a precise proportionality of excitation and inhibition (Sanchez-Vives and McCormick, 2000; Compte et al., 2003; Shu et al., 2003). In departure from the "balanced input hypothesis," our results show that in the absence of any network activity, strong glutamatergic stimulations regularly generate dendritic regenerative plateau potentials capable of driving the somatic membrane into a stable long-lasting depolarized state. Our measurements performed in the sodium channel blocker tetrodotoxin (TTX) show that AP-mediated IPSPs are not the only mechanism for the maintenance of the depolarized equilibrium state in pyramidal neurons.

Recently, two groups (Schiller et al., 2000; Oakley et al., 2001) showed that the input-output function of basal dendrites is characterized by a step transition ("spike") at some critical glutamate level. Although these experiments revealed an important feature of basal branches, the evoked somatic depolarizations were subthreshold for generation of the somatic APs, and therefore the link between basal dendritic spikes and UP state-like depolarizations had not been established. In our experiments, single gluta- 
mate pulses (duration, $5 \mathrm{~ms}$ ) delivered on single basal dendrites invariably produce long-lasting somatic plateau depolarizations (duration, 70-500 ms), accompanied by bursts of somatic APs. This characteristic electrical signal, depolarization plateau combined with somatic sodium spikes, resembles in vivo UP states in several aspects, including (1) the amplitude of the depolarization, (2) the duration of the plateau phase, and (3) intermittent firing during the plateau event (Milojkovic et al., 2004). The results presented here demonstrate that prefrontal pyramidal neurons readily switch to the activated (UP) state after suprathreshold stimulation of a single basal dendrite and that the initiation and maintenance of a stable period of activity in vitro is based on the dendritic physiological properties.

\section{Materials and Methods}

Electrophysiology and morphology. Coronal slices $(300 \mu \mathrm{m})$ anterior to genu of corpus callosum were harvested from Sprague Dawley rats (postnatal days 21-42) according to an animal protocol approved by the Yale University Animal Care and Use Committee. Animals were anesthetized with halothane and decapitated, and the brain was removed with the head immersed in ice-cold artificial CSF (ACSF). The ACSF contained (in mM) $125 \mathrm{NaCl}, 26 \mathrm{NaHCO}_{3}, 10$ glucose, $2.3 \mathrm{KCl}, 1.26 \mathrm{KH}_{2} \mathrm{PO}_{4}, 2$ $\mathrm{CaCl}_{2}$, and $1 \mathrm{MgSO}_{4}$, $\mathrm{pH}$ 7.4. TTX $(1 \mu \mathrm{M})$ was purchased from Sigma (St. Louis, MO). Whole-cell recordings were made at $29-33^{\circ} \mathrm{C}$ from visually identified layer $\mathrm{V}$ and layer II/III pyramidal neurons on the medial and dorsomedial part of the slice. The intracellular solution contained (in mM) $135 \mathrm{~K}$-gluconate, $2 \mathrm{MgCl}_{2}, 3 \mathrm{Na}_{2}$-ATP, $10 \mathrm{Na}_{2}$-phosphocreatine, 0.3 $\mathrm{Na}_{2}$-GTP, and 10 HEPES, pH 7.3 adjusted with $\mathrm{KOH}$. Electrical signals were amplified with Multiclamp 700A and digitized with two input boards: (1) Digidata Series 1322A (Axon Instruments, Union City, CA) at a $5 \mathrm{kHz}$ sampling rate and (2) Neuroplex (RedShirtImaging, Fairfield, $\mathrm{CT})$ at a $1 \mathrm{kHz}$ sampling rate. Only cells with a membrane potential more hyperpolarized than $-55 \mathrm{mV}$ and $\mathrm{AP}$ amplitudes $>80 \mathrm{mV}$ (measured from baseline) were included in this study. Electrophysiological profiles of pyramidal neurons were assessed using the progressive increases in the amplitude of the depolarizing current pulses injected into the soma (Yang et al., 1996). To allow positioning of glutamate stimulation electrodes, neurons were filled through whole-cell recording pipettes with either rhodamine-dextran $3000(80-100 \mu \mathrm{M})$ or voltage-sensitive dyes (see below), dissolved in standard K-gluconate-based intracellular solution. After the experiment, fluorescence images were taken with a CCD camera (Dage IR-1000) on an Axioskop 2FS (Zeiss, Thornwood, NY). Some neurons were detached from the recording pipette (outside-out patch), transferred onto the microscope slide, and mounted temporarily in a drop of ACSF. Photographs of fluorescent neurons were made on a Zeiss Axiophot microscope equipped with an AxioCam HRc camera. Twenty-one of 48 neurons, in which the stimulation pipette was positioned blindly (sniffing for dendrites), were filled with biocytin $(0.5 \%)$ and processed with an avidin-based kit (Vectastain PK-6100 Standard; Vector Laboratories, Burlingame, CA).

Optical measurements. Voltage-sensitive dye recordings were essentially as described previously (Antic, 2003). Briefly, the voltage-sensitive dye JPW3028 was obtained from L. M. Loew and J. P. Wuskell (University of Connecticut Health Center). JPW1114 was purchased from Molecular Probes (Eugene, OR). Dyes were dissolved in intracellular solution $(0.8-1.5 \mathrm{~mm})$ and loaded into neurons through whole-cell pipettes $(\sim 30 \mathrm{~min})$. Loading pipettes were then pulled out (outside-out patch), and neurons were incubated at room temperature for $1-2 \mathrm{~h}$. Before optical recordings, neurons were transferred to a recording microscope equipped with a NeuroCCD camera (RedShirtImaging) and repatched with dye-free electrodes. Dendrites were illuminated with a xenon $250 \mathrm{~W}$ arc lamp (770 X W/T; Opti-Quip, Highland Mills, NY). Optical signals (excitation, $520 \pm 45 \mathrm{~nm}$; dichroic, $570 \mathrm{~nm}$; emission, $>610 \mathrm{~nm}$ ) were recorded with $80 \times 80$ pixels at a $1 \mathrm{kHz}$ frame rate, stored, and temporally filtered (off-line) with a digital Gaussian low-pass filter (150 Hz cutoff) and a Butterworth high-pass filter $(0.4 \mathrm{~Hz})$, unless specified otherwise. To correct for photobleaching, the trace without stimulus was recorded at the end of the experiment and subtracted from the traces shown in Figures 6 and 8 . The term "region of interest" (ROI) was used to mark selected neuronal compartments, where membrane potential transients were measured either optically (voltage-sensitive dyes) or electrically (whole-cell).

Glutamate application. Sharp $(40 \pm 10 \mathrm{M} \Omega)$ pipettes were pulled from borosilicate glass with filament ( $1.5 \mathrm{~mm}$ outer diameter) and backfilled with $200 \mathrm{~mm}$ Na-glutamate, $\mathrm{pH}$ 9. A programmable stimulator, Master-8, and a stimulus isolation unit, IsoFlex (A.M.P.I., Jerusalem, Israel), were used to iontophoretically eject glutamate. A motorized micromanipulator, Sutter MP-285 (Sutter Instrument, Novato, CA), was used to drive the tips of glutamate pipettes diagonally ("fourth axis") into the slice tissue. The term "diagonal" is used throughout whenever both the $x$-axis and $z$-axis motors were engaged simultaneously. In experiments in which glutamate iontophoresis pipettes were positioned blindly, the following criteria were used to determine the adequate stimulation site. First, the tip of the stimulation pipette had to be $>70 \mu \mathrm{m}$ away from the cell body and $>30 \mu \mathrm{m}$ below the surface of the slice. Second, glutamate pulses of only $1.8 \mu \mathrm{A}$ ( $5 \mathrm{~ms}$ duration) had to trigger $>100$ ms plateau depolarizations accompanied by at least two APs (see Fig. 2D, traces 5-9). In some experiments depicted in Figure 3, glutamate was applied on basal dendrites as close as $50 \mu \mathrm{m}$ from the cell body. This was done because at distances $>80 \mu \mathrm{m}$ from the soma, the command voltage had to be set to values more negative than $-100 \mathrm{mV}$ to block the generation of glutamate-evoked plateaus.

Data analysis. Optical and electrical measurements were analyzed using the software Neuroplex 5.03 (RedShirtImaging) and Axoscope 8.1 (Axon Instruments). The plateau amplitude was measured as a difference between the peak depolarization after the last AP in the burst and at baseline. The duration of the plateau depolarization was measured at $50 \%$ of plateau amplitude (Fig. $2 \mathrm{D}$, right inset, dur.). The linear correlation coefficient, two-tailed Student's paired $t$ test (paired $t$ test), and graph plotting were done in Excel (Microsoft, Redmond, WA).

\section{Results}

\section{Focal glutamate stimulation}

In our experiments, glutamate was ejected iontophoretically from glass pipettes onto the visually identified basal dendrites buried deep inside the brain slice. The abundance of cellular membranes and tortuosity of the cortical neuropil impose a mechanical barrier for the diffusion of neurotransmitters (Franks et al., 2002). Enzymatic degradation and glutamate uptake (Matthews et al., 2000; Danbolt, 2001) further limit the spatial spread of glutamate ions from the tip of the glass pipette into the tissue. To determine how far glutamate can diffuse from the ejection site to influence the dendritic membrane, we performed the measurements described in Figure 1. Pyramidal layer V neurons were injected intracellularly with the fluorescent dye rhodamine, the tips of glutamate iontophoretic electrodes were first positioned $\sim 5 \mu \mathrm{m}$ from the shafts of visually identified basal dendrites (Fig. $1 A$ ), and the glutamate pipette was gradually retracted away from the dendrite. Before the beginning of the pipette withdrawal procedure, the intensity of the glutamate iontophoretic current was adjusted (usually in the range between 1.4 and $1.8 \mu \mathrm{A}$ ), so that a single 5-ms-long glutamate pulse triggered a somatic plateau potential (half-width, $>100 \mathrm{~ms}$ ) accompanied by two or more sodium APs. With the iontophoretic current fixed at this "suprathreshold" value, the glutamate pipette was retracted diagonally in $3 \mu \mathrm{m}$ steps. At each stop, a glutamate pulse was applied and somatic response was recorded with a whole-cell electrode (Fig. $1 B)$. A plot of the amplitude of somatic plateau depolarization obtained in 12 neurons (Fig. 1C) showed that diagonal displacement of the glutamate pipette $>12.4 \pm 5.8 \mu \mathrm{m}$ caused the stimulus to fail to trigger a regenerative plateau potential (Fig. $1 B$, red trace). Furthermore, diagonal displacement $>18.0 \pm 5.5 \mu \mathrm{m}$ re- 

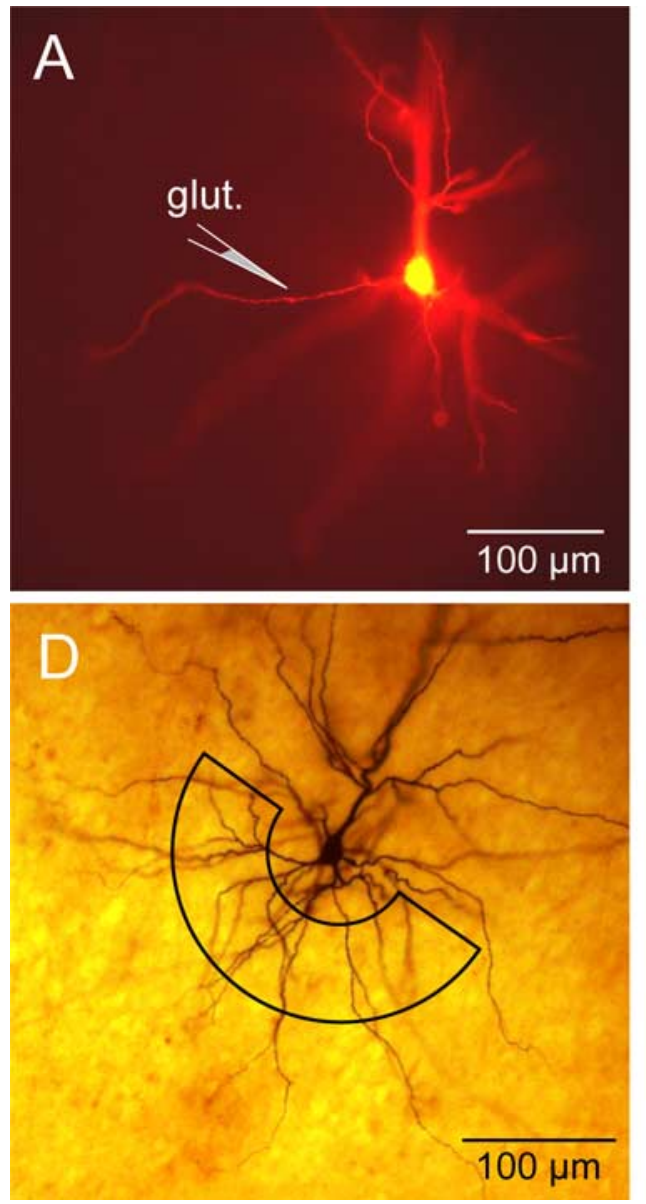
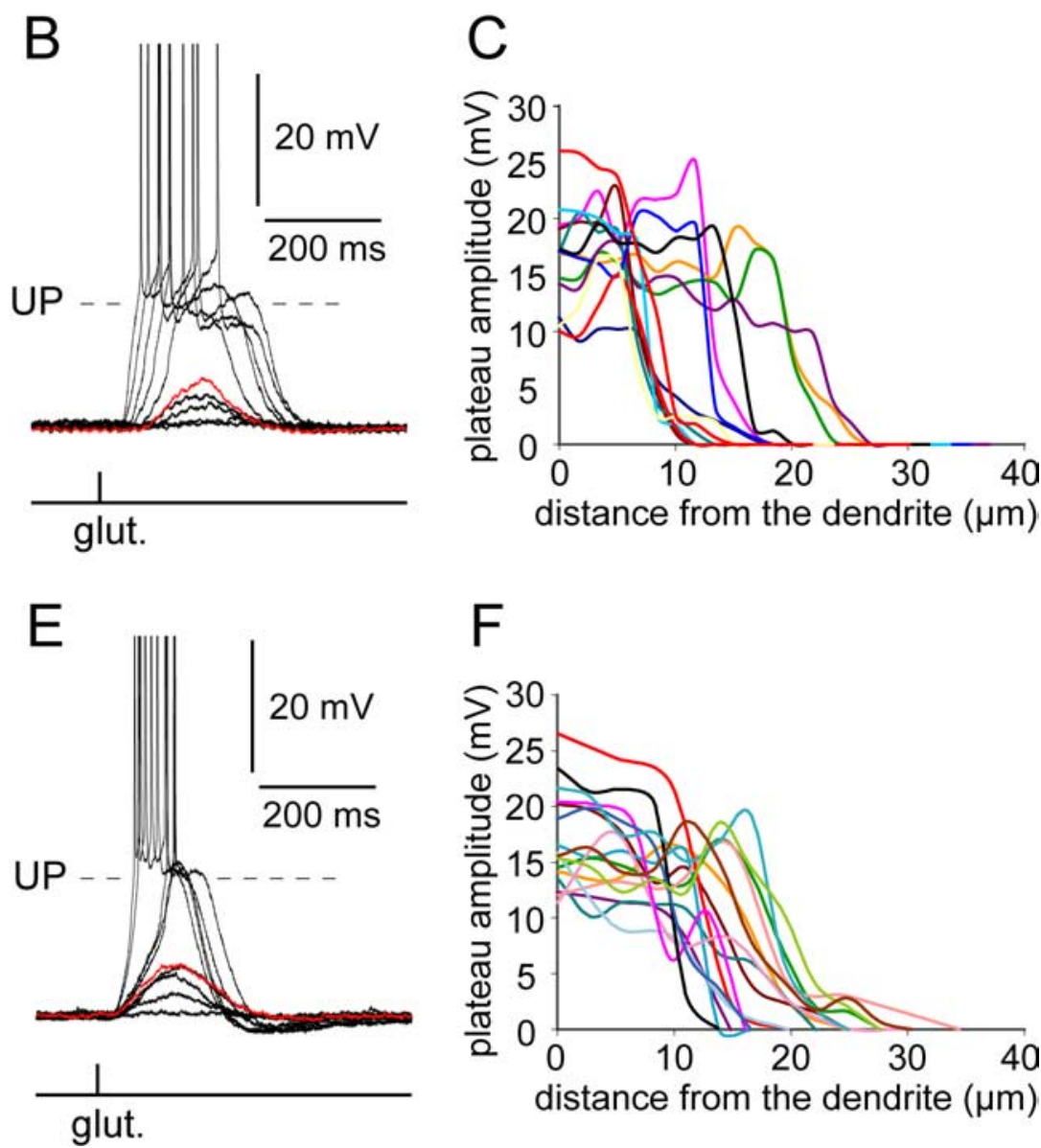
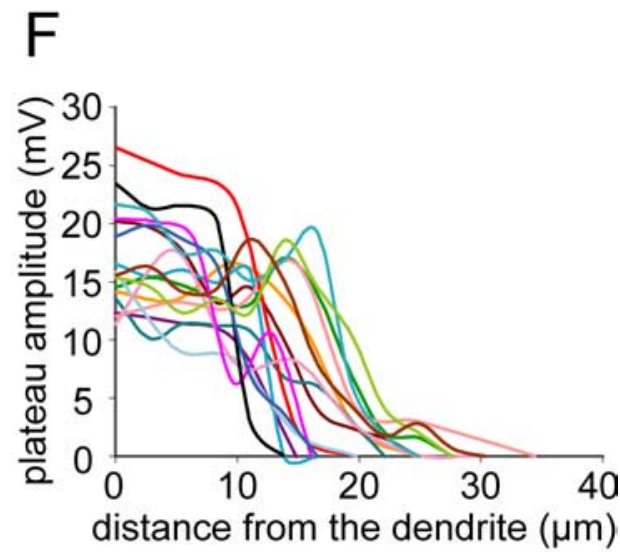

Figure 1. Spatial considerations of glutamate microiontophoresis. $\boldsymbol{A}$, Microphotograph of a rhodamine-filled layer $V$ pyramidal cell. The schematic drawing marks the position of the glutamate pipette $90 \mu \mathrm{m}$ from the center of the soma, $\sim 5 \mu \mathrm{m}$ from the dendritic shaft. $\boldsymbol{B}$. While keeping the stimulus parameters fixed (intensity, duration, and frequency), the glutamate pipette was withdrawn diagonally away from the basal dendrite in equal steps $(3 \mu \mathrm{m})$. At each stop, glutamate was iontophoretically ejected and the neuronal response was recorded by a whole-cell pipette. At a distance of $15 \mu \mathrm{m}$ from the dendrite, a previously suprathreshold glutamate pulse was no longer capable of triggering the UP state (red trace). $C$, The amplitude of the plateau depolarization is plotted versus the distance between the tip of the glutamate iontophoresis pipette and the dendritic shaft. At a distance of $\geq 27 \mu \mathrm{m}$, a previously suprathreshold glutamate pulse failed to evoke a detectable response (flat recording) in 12 of 12 neurons tested. $\boldsymbol{D}$, Microphotograph of 1 of 21 biocytin-stained layer $V$ pyramidal neurons obtained in experiments in which glutamate electrodes were positioned blindly (sniffing for dendrites). The black semicircular box marks the area around the cell body (70-120 $\mu$ m away from the center of the soma) in which glutamate stimulation sites were located. $\boldsymbol{E}$, A stimulation site found $50 \mu \mathrm{m}$ below the surface of the slice, $85 \mu \mathrm{m}$ from the center of the soma. Glutamate ejections (1.6 $\mu \mathrm{A}, 5 \mathrm{~ms}$ ) invariably produced UP states at $1 \mathrm{~Hz}$ stimulation frequency (data not shown). The tip of the glutamate pipette was then pulled away diagonally from the original stimulation site in increments of $3 \mu \mathrm{m}$, while the intensity and the duration of the iontophoretic current were kept unchanged. At $15 \mu \mathrm{m}$ from the "optimal" stimulation site, a previously suprathreshold amount of injected glutamate now failed to trigger an UP state (red trace). At a distance of $27 \mu \mathrm{m}$, glutamate pulses did not produce a detectable response (flat recording). $\boldsymbol{F}$, The plot of the amplitude of glutamate-evoked plateau versus the distance from the dendrite, obtained in 16 neurons. glut., Glutamate.

sulted in a complete failure of previously suprathreshold stimuli to evoke any measurable somatic depolarization-flat line $(n=$ 12). These results suggest that during brief ( $5 \mathrm{~ms}$ ) suprathreshold glutamate pulse, only a small circumscribed area of $\sim 25 \mu \mathrm{m}$ in radius is exposed to a detectable concentration of the exogenous neurotransmitter.

In the experiments described above, the positioning of the glutamate pipette onto basal dendrites was assisted by intracellularly applied fluorescent dye. These experiments established important technical details such as the resistance of the glass pipette, the concentration of intrapipette glutamate, and the characteristics of the iontophoretic pulse (intensity, duration, and frequency) required for initiation of UP state-like somatic depolarizations. It was soon realized that successful positioning of glutamate electrodes in the basilar dendritic tree could be done without the aid of fluorescent dyes, using so-called "sniffing for dendrites." While constantly applying iontophoretic pulses (1-2 $\mu \mathrm{A}, 5 \mathrm{~ms}, 2-4 \mathrm{~Hz}$ ), the glutamate pipette was advanced diago- nally into the slice tissue and the somatic membrane potential was monitored in current clamp. Between 30 and $80 \mu \mathrm{m}$ below the surface of the slice, and in the hemispherical space marked by the black line in Figure $1 D(70-120 \mu \mathrm{m}$ from the center of the soma), we sought and found glutamate stimulation sites that regularly produced characteristic somatic plateau depolarizations accompanied by bursts of APs (Fig. $1 E)(n=48$ of 48). In 16 neurons, we gradually retracted the stimulation pipette as described previously for rhodamine-filled cells. Glutamate-evoked depolarizations were again very sensitive to the withdrawal of the stimulation pipette (Fig. $1 F)$. At a critical distance (15.4 \pm 3.7 $\mu \mathrm{m})$ from the optimal stimulation site, glutamate ejections failed to trigger regenerative dendritic plateau potentials (Fig. $1 E$, red trace). At a distance of $22.8 \pm 6.0 \mu \mathrm{m}$, previously suprathreshold stimuli did not produce any measurable response (flat recording, $n=16$ ). Twenty-one of 48 neurons, in which positioning of the stimulating electrode was done by 
Table 1. All pyramidal cell types in the rat prefrontal cortex generate the plateau potentials after single glutamate pulses delivered on their basal dendrites

\begin{tabular}{|c|c|c|c|c|c|c|c|c|c|c|c|c|}
\hline & \multicolumn{3}{|c|}{ Intrinsic bursting } & \multicolumn{3}{|c|}{ Intermediate } & \multicolumn{3}{|c|}{ Regular spiking } & \multicolumn{3}{|c|}{ Oscillatory bursting } \\
\hline & $n$ & $\%$ & $V_{r}(m V)$ & $n$ & $\%$ & $V_{r}(m V)$ & $n$ & $\%$ & $V_{r}(\mathrm{mV})$ & $n$ & $\%$ & $V_{\mathrm{r}}(\mathrm{mV})$ \\
\hline Layer V & 20 & 62.5 & $59.8 \pm 3.7$ & 7 & 21.9 & $61.4 \pm 6.1$ & 5 & 15.6 & $58.1 \pm 2.7$ & 0 & 0.0 & \\
\hline Layer II/III & 7 & 58.3 & $64.3 \pm 4.9$ & 2 & 16.7 & $68.0 \pm 1.4$ & 2 & 16.7 & $63.1 \pm 2.6$ & 1 & 8.3 & 68 \\
\hline
\end{tabular}

$n$, Number of cells; $\%$, percentage of the total sample; $V_{r}$, resting membrane potential (mean \pm SD).

sniffing, were stained with biocytin and confirmed to be layer V pyramidal neurons (Fig. 1D).

\section{Layer and cell-type nonspecificity}

To test whether different pyramidal neuron cell types can generate the plateau potentials, in one group of neurons we analyzed the firing pattern using the progressive increases in the amplitude of the direct current depolarization (Yang et al., 1996). In all 32 layer $\mathrm{V}$ pyramidal neurons and in all 12 layer II/III pyramidal neurons analyzed in this way (Table 1), brief glutamate pulses delivered on the basal dendrites evoked sustained depolarization of the cell body. We found only one layer II/III pyramidal cell that exhibited rhythmic oscillatory bursting (supplemental Fig. S1 Ad, available at www.jneurosci.org as supplemental material). However, numerous recordings from intrinsic bursting, intermediate, and regular spiking types in both layer V and layer II/III (Table 1) suggested that plateau generation is not specific to any subclass of pyramidal neurons.

\section{Input-output function}

We next studied how neuronal output (somatic depolarization and AP firing) depended on the magnitude of the glutamatergic input delivered onto the basal dendrites? The tips of the glutamate stimulation pipettes were positioned $5-10 \mu \mathrm{m}$ from rhodamine-filled dendrites, $70-110 \mu \mathrm{m}$ away from the cell body (Fig. 2C). The intensity of the iontophoretic current was increased gradually, and the somatic membrane potential was monitored in current clamp (Fig. 2D). To prevent sensitization or desensitization of glutamate receptors, a $3 \mathrm{~s}$ waiting period was allowed between glutamate ejections. The plot of the amplitude of somatic plateau depolarization showed a step transition at some critical intensity of glutamate stimulation (Fig. 2D, left inset, red trace). Additional increase in the glutamate current intensity did not contribute significantly to the amplitude of the somatic plateau (Fig. $2 E$ ). In contrast, the duration (half-width) of the somatic plateau phase, as well as the number of APs per plateau event, increased gradually, in a linear manner, with the intensity of the glutamate current (Fig. $2 F, G$ ). All neurons tested in this way $(n=15)$ showed a bimodal distribution of somatic depolarizations (supplemental Fig. S2 A, available at www. jneurosci.org as supplemental material). With an exception of the narrow stimulus intensity range, centered around the critical glutamate concentration (1 $\mu \mathrm{A})$ (supplemental Fig. S2 $B$, available at www.jneurosci.org as supplemental material), neurons were found either in a resting state $(\mathrm{DOWN} ; 0 \mathrm{mV})$ state or in a depolarized (UP) state $(\sim 15 \mathrm{mV}$, relative to resting membrane potential). For instance, the mean amplitude of the depolarized state measured in 15 neurons at the three different intensities of the glutamate iontophoretic currents $1.6,1.8$, and $2.0 \mu \mathrm{A}$ was $15.4 \pm 2.5,15.5 \pm 3.3$, and $15.8 \pm 2.8 \mathrm{mV}$, respectively (for the average values of neuronal responses to other glutamate current intensities, see supplemental Fig. S2 B, available at www.jneurosci. org as supplemental material). Experiments with gradually increasing glutamate iontophoretic currents show that the inputoutput function of basal dendrites is characterized by hard threshold followed by saturation of the response. Once the critical glutamate concentration for the generation of the plateau potential is reached, an additional increase in stimulus intensity is interpreted by an increase in the duration of the plateau depolarization (Fig. $2 \mathrm{~F}$; supplemental $\mathrm{S} 2 \mathrm{C}$, available at www.jneurosci.org as supplemental material).

\section{Voltage-dependent nature of glutamate-evoked plateaus}

The results presented in Figure 2 suggested that glutamateevoked dendritic potentials were regenerative in nature (all-ornone membrane potential transients with fixed maximal amplitude but variable duration). To test this hypothesis, in the next series of experiments, the stimulation pipette was positioned on individual basal dendrites $(50-80 \mu \mathrm{m}$ from the soma), and glutamate-evoked responses were monitored in voltage clamp (Fig. 3). Glutamate ejections regularly caused postsynaptic inward currents, the characteristics of which resembled the somatic plateau potentials described in Figure 2. Namely, these inward currents exhibited a sharp onset, a plateau phase lasting between 27 and $189 \mathrm{~ms}$, and abrupt collapse at the end of the plateau $(n=$ 8). In six of eight neurons, a fast spikelet was recorded at the very beginning of the plateau phase (Fig. $3 A$, arrow). When command voltage was shifted gradually in the hyperpolarizing direction, the interval between the glutamate injection and onset of the inward current (latency) increased gradually, whereas the duration of the plateau phase decreased (Fig. 3E). At some very negative command potential, a previously suprathreshold glutamate pulse failed to trigger a fast-rising square-shaped inward component (Fig. 3A, trace 5). The glutamate-evoked inward current, however, did not go to zero. A slow-rising "voltage-insensitive" remnant current was present in eight of eight neurons tested (Fig. 3A, trace 5). It appears that glutamate-evoked inward current is composed of two distinguishable components. A voltage-sensitive fast square-shaped component (Fig. 3B, -58) is riding on the top of the smaller and slower voltage-insensitive depolarizing current foot $(-82)$. The time course of the "foot" component resembles the time course of the subthreshold membrane potential changes observed in the input-output function obtained in current clamp (Fig. 2D, left inset, trace 4). A plot of the peak amplitude of the glutamate-evoked inward current versus membrane voltage showed a clear step transition at some critical membrane potential (Fig. 3D, arrowhead). Altogether, these data indicate that glutamate concentrations required to produce a sustained somatic depolarization during the in vitro-evoked UP state (Fig. $3 A$, trace 0 ) actually triggered an active membrane response (spike), characterized by fast onset, long-lasting plateau and abrupt breakdown.

\section{Experiments with sodium channel blocker TTX}

Basal dendrites of cortical pyramidal neurons are endowed with TTX-sensitive dendritic sodium currents (Schiller et al., 1995). One would expect that strong postsynaptic depolarizations (such as those described in Fig. 2) would readily activate dendritic sodium conductances. Depending on the density of voltage-gated sodium channels, glutamate-evoked depolarizations are ex- 


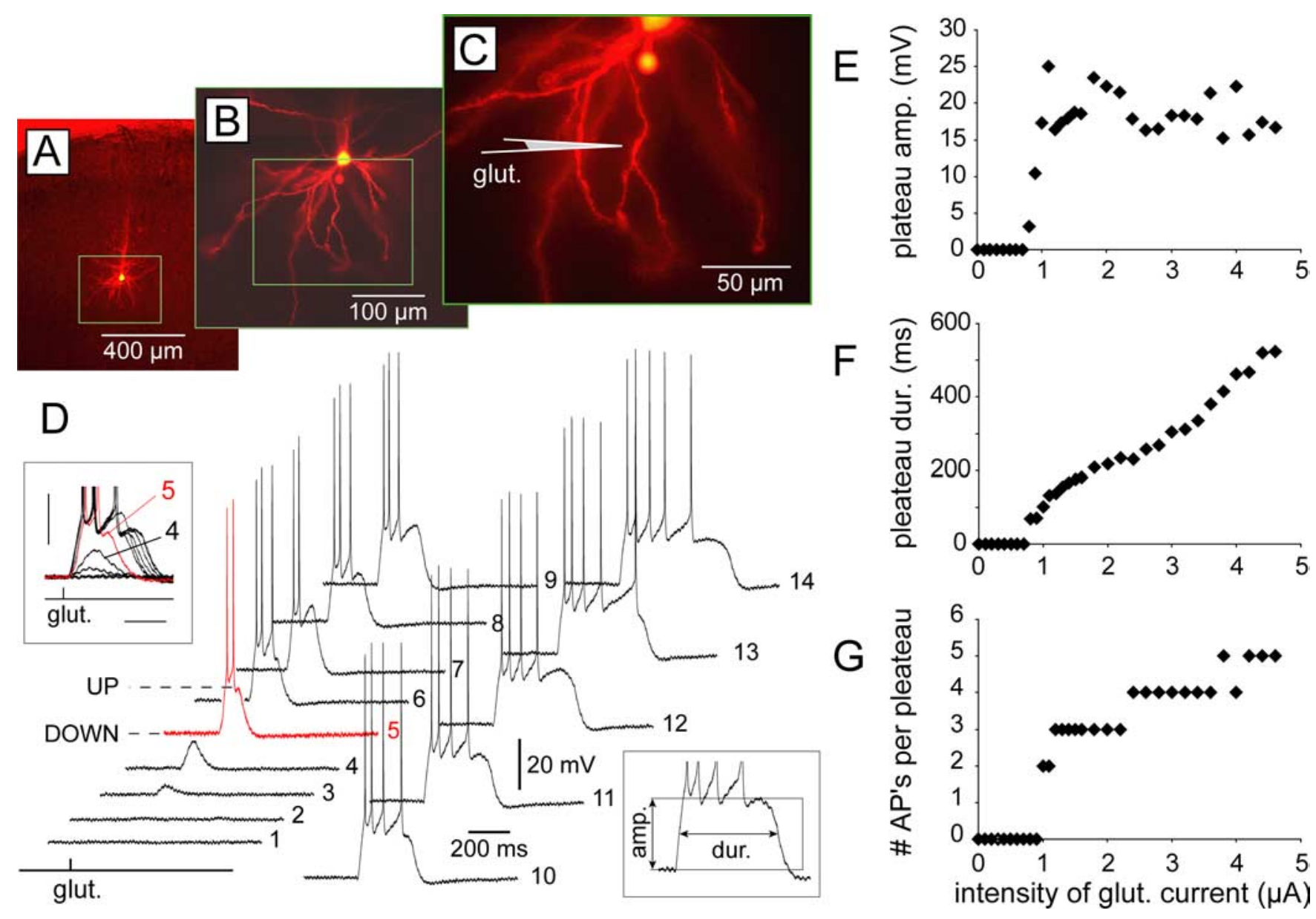

Figure 2. Input- output function of basal dendrites-somatic whole cell. $A$, A coronal slice cut from the rat medial prefrontal cortex was illuminated simultaneously with infrared and excitation light to show the position of the rhodamine-filled neuron with respect to the pia. $\boldsymbol{B}$, An area indicated by the rectangle in $\boldsymbol{A}$ is increased to show morphological details of the basal dendritic tree. $C$, An area indicated by the rectangle in $\boldsymbol{B}$ is increased to show the segment of the basal dendrite (85 $\mu \mathrm{m}$ away from the soma) where a glutamate-filled glass pipette (schematic drawing) was positioned during the recordings shown in $\boldsymbol{D}$. D. The tip of glutamate iontophoretic pipette was kept in a fixed position, whereas the intensity of the glutamate current $\left(I_{\mathrm{g}}\right)$ was increased gradually from 0 to $1.6 \mu \mathrm{A}$ in increments of $0.1 \mu \mathrm{A}$ and from 1.8 to $4.6 \mu \mathrm{A}$ in increments of $0.2 \mu \mathrm{A}$. Fourteen of 32 steps are displayed. In this and the following figures, "glut." marks the timing of the glutamate pulse (duration, $5 \mathrm{~ms}$ ). Left inset, Eleven successive sweeps (glutamate current intensities, $0.6-1.6 \mu \mathrm{A}$ ) are superimposed to show the transition between the subthreshold (sweep 4; $I_{\mathrm{g}}=0.9 \mu \mathrm{A}$ ) and suprathreshold (red sweep $5 ; I_{\mathrm{g}}=1.0 \mu \mathrm{A}$ ) stimuli. Right inset, Plateau amplitude was measured as a difference between the peak depolarization after the last AP in the burst and at the baseline (amp.). In trials in which the neuron did not generate any APs (e.g., sweeps 3 and 4), the amplitude of the peak depolarization was plotted in $E$. Duration of the plateau depolarization was measured at $50 \%$ of plateau amplitude (dur.). Vertical scaling applies for all traces in $\boldsymbol{D}$, including those in the insets. The horizontal calibration bar in the left inset is $100 \mathrm{~ms}$. The somatic response to glutamate stimulation was analyzed in terms of plateau amplitude $(\boldsymbol{E})$, plateau duration $(\boldsymbol{F})$, and number of APs per plateau event $(\boldsymbol{G})$.

pected to either trigger dendritic regenerative potentials (Rhodes, 1999; Archie and Mel, 2000; Ariav et al., 2003) or at least provide some boosting of the postsynaptic signal (Stuart and Sakmann, 1995). Therefore, if sodium currents do contribute to the glutamate-evoked depolarization, then block of sodium channels should result in a lesser amplitude of the postsynaptic signal. In contrast, both the amplitude and the half-width of the glutamateevoked somatic plateau appeared to be greater in TTX than in the control condition (Fig. 4B). The mean plateau amplitudes in control and TTX conditions were $16.6 \pm 3.5$ and $22.4 \pm 3.9 \mathrm{mV}$, respectively $(n=14)$. Although the increase in amplitude was very significant (paired $t$ test; $p<0.00001$ ), the statistical significance of the TTX-induced increase in the duration (from $296 \pm$ 101 to $311 \pm 94 \mathrm{~ms})$ was not so prominent $(p=0.01790 ; n=14)$. The mean percentage of increase in amplitude and duration was $38.5 \pm 24.2$ and $9.3 \pm 20.6 \%$, respectively (Fig. $4 D$ ). These data suggest that the actual magnitude of the glutamate-evoked somatic depolarization in a bursting neuron is partially masked by AP afterhyperpolarization. The removal of sodium spikes (by TTX) revealed the true magnitude and temporal dynamics of the dendrite-generated current that reached the soma and caused the depolarization of the somatic membrane. After a single pulse (duration, $5 \mathrm{~ms}$ ) exposure to an excitatory neurotransmitter (Fig. $4 B$, glut.), pyramidal neurons generated a plateau potential that outlasted the stimulus $\sim 100$ times over. In the absence of any AP-dependent (network-driven) feedback inhibition, the somatic membrane was experiencing a sustained depolarization (Fig. 4C, TTX), in 14 of 14 neurons tested.

\section{Correlation between dendritic and somatic plateau depolarizations}

In the experiments presented above, the dendritic function was studied indirectly, using the somatic whole-cell pipettes. Dendritic membrane potentials are known to undergo drastic transformations on the way to the cell body. For instance, there is hardly any resemblance between membrane potential waveforms recorded in distal apical dendrites and those obtained from the cell body (Larkum et al., 2001). To characterize glutamateevoked membrane potential transients in basal dendrites, in the next series of experiments we used simultaneous whole-cell and 

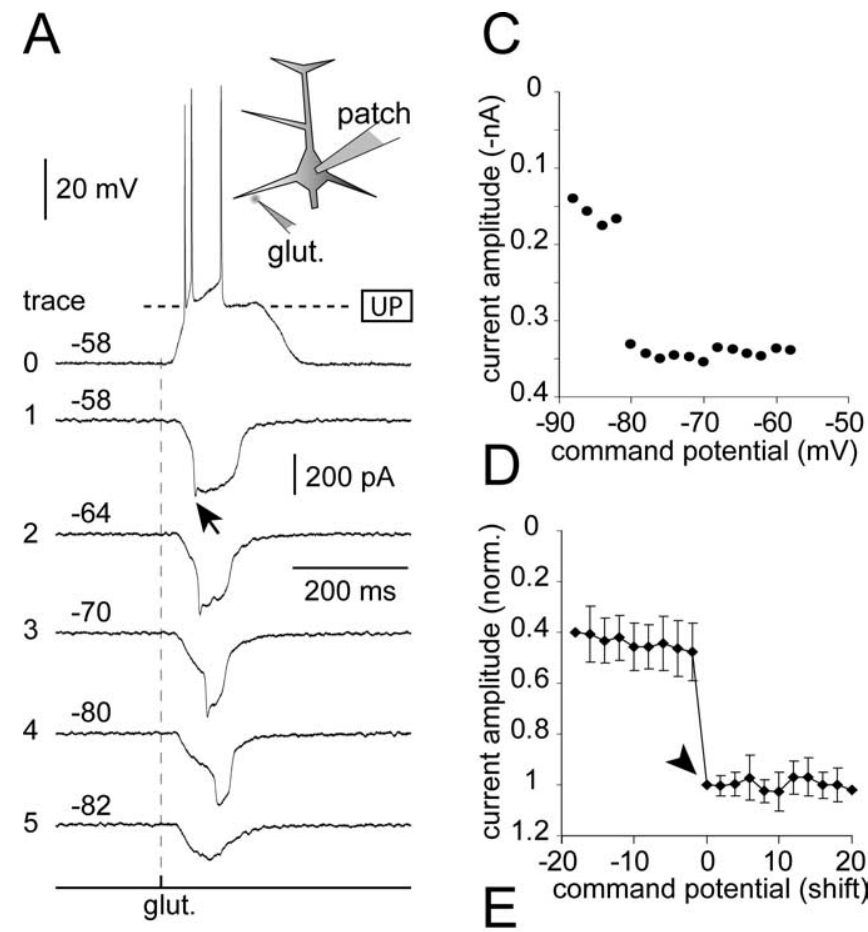

B

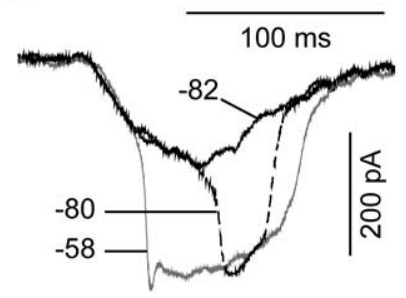

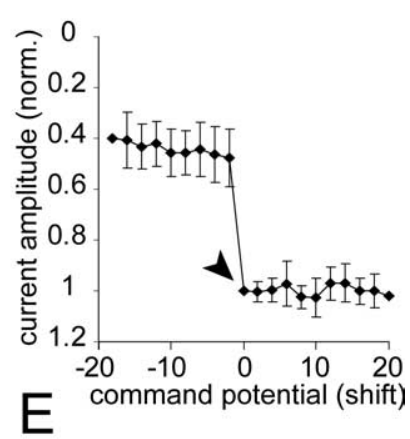
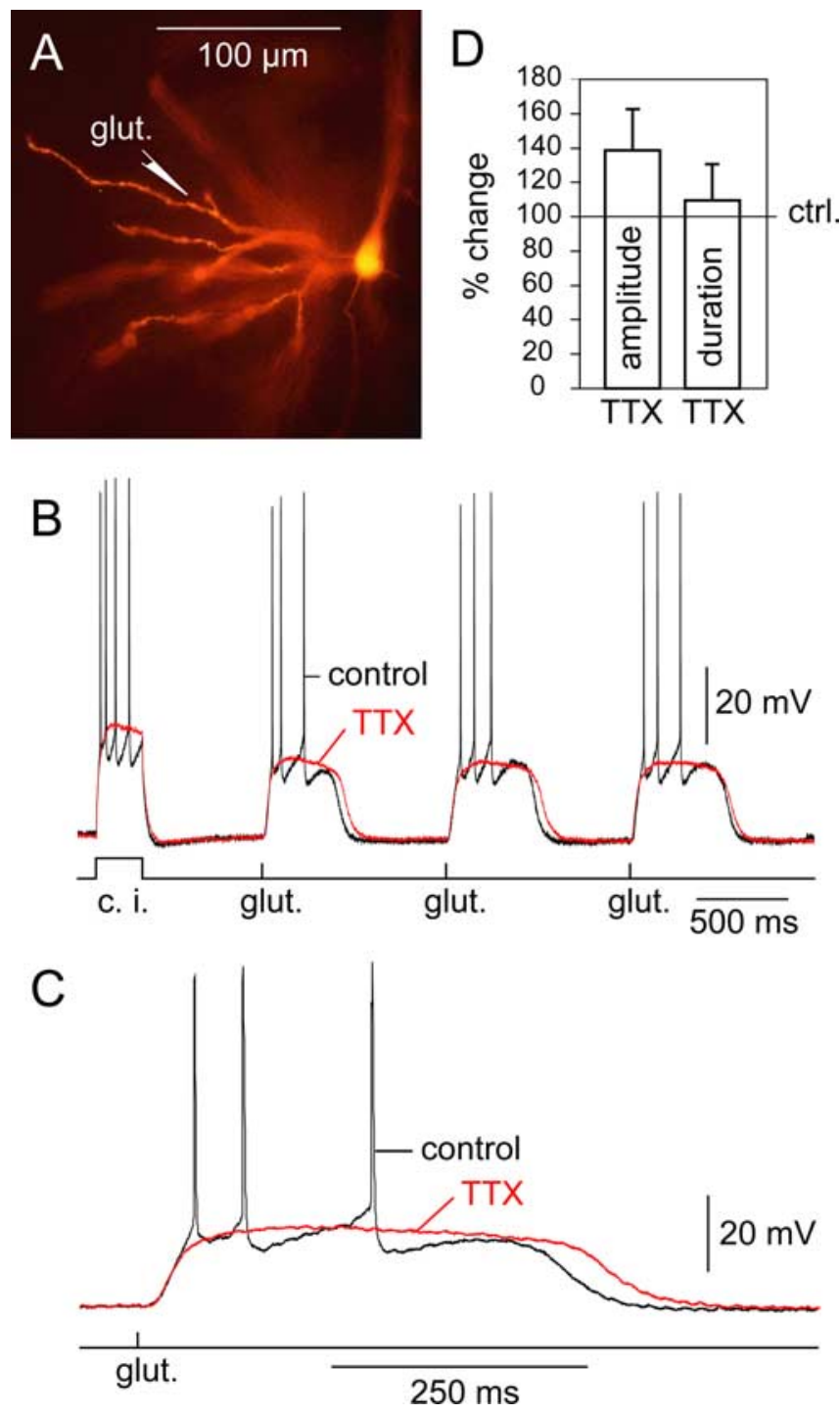

Figure 3. Regenerative properties of glutamate-evoked plateau potentials. $\boldsymbol{A}$, A glutamate (glut.)-filled pipette was positioned on a basal dendrite (75 $\mu \mathrm{m}$ from the center of the soma), as indicated in the schematic drawing (inset). Trace 0 , Current-clamp recording of the evoked UP state. Traces $1-5$, While keeping the intensity $(1.5 \mu \mathrm{A})$ and duration $(5 \mathrm{~ms})$ of the iontophoretic current pulse fixed between trials, the recording configuration was changed to voltage clamp. Glutamate-evoked postsynaptic currents were recorded at different command potentials (from -58 to $-88 \mathrm{mV}$, in steps of $2 \mathrm{mV}$ ). Five of 16 voltage-clamp sweeps are selected for display (traces 1-5). The arrow marks an initial spikelet that precedes the plateau phase. $\boldsymbol{B}$, Three characteristic traces are selected from $A$ and superimposed on a faster time scale to show (1) the voltage-dependent change in the duration of the evoked spike (traces -58 and -80$),(2)$ the residual (foot) current component ( -82 ), and ( 3 ) an abrupt transition between -80 and -82 $\mathrm{mV}$. C, The absolute amplitude of the plateau phase of the glutamate-evoked inward current is plotted versus the command potential, for the experiment shown in $\boldsymbol{A}$. $\boldsymbol{D}, \mathrm{A}$ plot of mean normalized amplitudes obtained in eight neurons. The command potential at which the steplike transition occurred is plotted as a zero $(0 \mathrm{mV})$. 0 ther command potentials in the corresponding data set are shifted accordingly. All data points, within a neuron, are first normalized with respect to the amplitude of inward current at the transition point (arrowhead) and then averaged across neurons. $\boldsymbol{E}$, Same as in $\boldsymbol{D}$, except instead of amplitude we plotted the duration (half-width) of the spike. The values are normalized with respect to the longest duration in each data set. The error bars in $\boldsymbol{D}$ and $\boldsymbol{E}$ are SDs $(n=8)$. norm., Normalized.

multisite voltage-sensitive dye imaging (Fig. 5). Neurons were first filled internally with the fluorescent voltage-sensitive dye JPW3028 or JPW1114, and then glutamate iontophoretic pulses (duration, $5 \mathrm{~ms}$; intensity, 1-3 $\mathrm{AA}$ ) were delivered on visually identified basal dendrites. The tips of glutamate-filled glass electrodes were positioned 70-150 $\mu \mathrm{m}$ away from the center of the cell body (mean $\pm \mathrm{SD}, 113.0 \pm 23 \mu \mathrm{m}$ ) and 5-10 $\mu \mathrm{m}$ from the
Figure 4. Effects of the sodium channel blocker TTX on glutamate-evoked plateau depolarizations. $A$, Composite microphotograph of a layer V pyramidal cell stained with rhodamine. The schematic drawing marks the position of the glutamate pipette $90 \mu \mathrm{m}$ from the soma. $\boldsymbol{B}$, Whole-cell recordings of glutamate-evoked somatic depolarizations in control (normal ACSF) and TTX solution (1 $\mu \mathrm{m}$ ). c.i., Current injection (200 pA, $250 \mathrm{~ms}$ ); glut., iontophoretic ejection of glutamate $\left(I_{g}=2.0 \mu \mathrm{A}\right.$; duration, $5 \mathrm{~ms}$; frequency, $\left.1 \mathrm{~Hz}\right)$. $C$, One section from $\boldsymbol{B}$ is displayed at a faster time scale to show the characteristics of the plateau phase. $\boldsymbol{D}$, Average percentage of change in the amplitude and duration of the somatic plateau depolarization $(n=14)$. ctrl., Control. The error bars are SDs.

dendritic shaft. A single glutamate pulse (duration, $5 \mathrm{~ms}$ ) was delivered per each recording sweep.

Voltage-sensitive dye measurements revealed that basal dendrites of pyramidal neurons respond to glutamate stimulation with characteristic potentials. These potentials were characterized with a fast upstroke, a long-lasting plateau phase, and an abrupt breakdown at the end of the plateau. In the group of neurons studied with voltage-sensitive dyes $(n=21)$, there was no statistical difference between the half-width of the glutamateevoked plateau potential in the target dendrite (Fig. 5B, T-dend.) and the half-width of the somatic plateau depolarization (Student's paired $t$ test; $p=0.6992 ; n=21$ ). The mean half-widths of dendritic and somatic plateau depolarizations were $302.9 \pm$ 106.2 and $301.1 \pm 103.4 \mathrm{~ms}$, respectively (range, 147-545 ms). The mean ratio of dendritic and somatic plateau half-width (den- 
drite/soma $\times 100 \%)$ was $99.9 \pm 7.5 \%$ (Fig. 5D). In all neurons, the onset of the somatic plateau was preceded by the onset of the glutamate-evoked dendritic spike. An average, latency of the somatic signal measured at half-amplitude of the slow component (supplemental Fig. S3, inset, available at www.jneurosci.org as supplemental material) was $8.2 \pm 2.6 \mathrm{~ms}(n=$ 21). A characteristic somatic plateau depolarization (amplitude, $>12 \mathrm{mV}$; duration, $>100 \mathrm{~ms}$ ) was never observed in the absence of the fast-rising square-shaped dendritic plateau potential.

Slow somatic depolarizations have been found recently to follow AP burst discharges in neocortical pyramidal neurons. These "delayed depolarizations" were attributed to AP-assisted calcium electrogenesis in the apical trunk (Larkum et al., 1999; Schwindt and Crill, 1999). Therefore, we tested whether AP-activated apical membrane conductances were responsible for the generation and maintenance of the sustained somatic depolarization in neurons with basal dendrites that were exposed to suprathreshold glutamatergic stimulation. In seven neurons, a negative current pulse was injected into the cell body to prevent the firing of sodium spikes (Fig. 6D). The intensity of the injected current was adjusted to a minimal value required to just block the generation of somatic APs. These negative current pulses did not prevent the generation of the dendritic plateau potentials far away from the cell body (Fig. 6D, ROI 4), nor did they block the genesis of plateau depolarizations in the soma (ROI 0). There was no difference between the duration of dendritic and somatic signal in the absence of APs (Student's paired $t$ test; $p=$ 0.8096; $n=7)$.

These experiments eliminated the possibility that AP-activated transmembrane currents in the apical trunk were solely responsible for plateau depolarizations observed after suprathreshold glutamatergic stimulations of the basal dendritic tree. Our experiments have not only ruled out the apical trunk as a major contributor of the depolarizing current, they successfully pinpointed the actual basilar branch that experienced the fastest and strongest depolarization. One advantage of voltage-sensitive dye measurements is the ability to monitor dendritic membrane potential transients in several neighboring dendrites at the same time. Multisite recordings revealed, in a most direct way, that the source of the depolarizing wave responsible for generation of the somatic plateau was actually in the basal dendrite exposed to the glutamate pulse (target dendrite). Strong depolarization transients were recorded all along the target dendrite: at the glutamate stimulation site (Fig. 6C, ROI 4) as well as in the more proximal regions of the same dendrite (ROI 3). Neighboring nontarget basal branches (Fig. 6C, ROIs 1 and 2) were experiencing APs that propagated from the cell body back into the basal dendrites (Antic, 2003). Optical measurements performed in proximal regions of nontarget basal branches also detected small long-lasting square-shaped depolarizations, which were often superimposed with backpropagating APs (BAPs). Based on the slower time course and smaller relative amplitude of the voltage-sensitive dye signal (Figs. $5 B, C, 6 C, D$, $7 C$ ), the depolarizing potentials in nontarget dendrites were attributed to a centrifugal spread of the somatic plateau signal into the periphery. Similar to APs, somatic plateau depolarizations also travel from the cell body into inactive basal dendrites. The relative depolarizations caused by backpropagating plateau potentials (BPPs) were much smaller than those caused by BAPs. However, with respect to duration of the signal, BPPs had a considerable advantage over BAPs, because BBPs lasted throughout the entire duration of the neuronal UP state $(70-500 \mathrm{~ms})$. It can be said that synaptic contacts concentrated in proximal portions of inactive (nontarget) dendrites oscillate in sync with the somatic plateau depolarizations during evoked UP states in vitro.

\section{Dendritic potentials in TTX-treated neurons}

In the next group of experiments, TTX was introduced in the bath and glutamate-evoked dendritic plateau potentials were recorded 

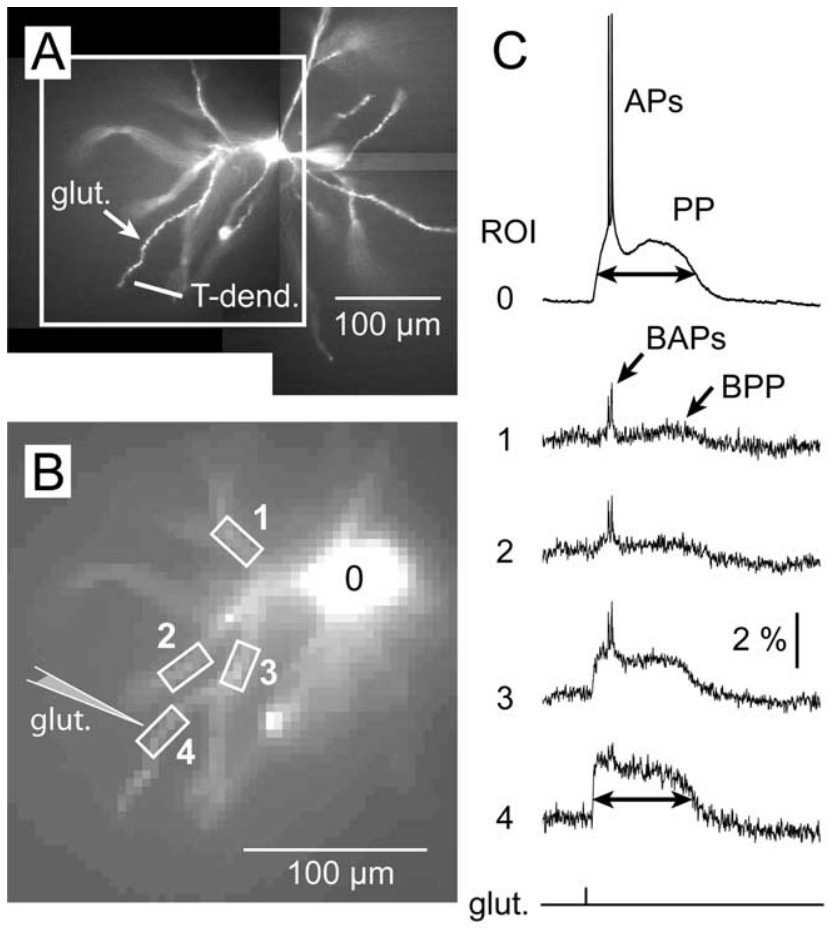

hyper.

Figure 6. Sustained somatic depolarizations originate in basal dendrites. $A$, Composite microphotograph of a layer V pyramidal cell injected with the voltage-sensitive dye JPW3028. The schematic drawing indicates the position of the glutamate-filled pipette (160 $\mu \mathrm{m}$ from the center of the soma). T-dend., Target dendrite, the dendrite exposed to glutamate pulse. $\boldsymbol{B}$, An area indicated by the rectangle in $\boldsymbol{A}$ is captured by an $80 \times 80$ pixel acquisition camera. The boxes mark four Rols where optical signals were selected for spatial averaging and display. C, A characteristic electrical signal, composed of two APs superimposed with the somatic plateau potential (PP) was evoked with a single glutamate pulse (duration, $5 \mathrm{~ms} ; I_{\mathrm{g}}=1.8 \mu \mathrm{A}$ ). Voltage-sensitive dye recordings obtained simultaneously from four ROls (ROls $1-4$ ) are aligned with the whole-cell somatic recording (ROI 0$)$. The double-headed arrow indicates the half-width of the plateau depolarization. $\boldsymbol{D}$, Same as in $\boldsymbol{C}$, except a negative current pulse (hyper.) was injected into the cell body to suppress firing. glut., Glutamate.

using voltage-sensitive dyes (Fig. 7). In all neurons tested $(n=9)$, the time course of the somatic signal (Fig. 7C, ROI 0) was found to be a faithful copy of the membrane potential change in the target dendrite (ROI 1). Both the dendritic and somatic voltage signals exhibited a fast onset, a plateau phase, and an abrupt collapse at the end of the plateau phase. In TTX-treated neurons, the duration (half-width) of the somatic plateau potential (300 \pm $88 \mathrm{~ms}$ ) was in a strict correlation with the duration of the dendritic depolarization ( $289 \pm 83 \mathrm{~ms}$; paired $t$ test; $p=0.3208 ; n=9)$.

At this point, it is important to note that the intracellularly applied voltage-sensitive dyes JPW3028 and JPW1114, although useful for monitoring the time course of the membrane potential change in neocortical pyramidal neurons (Antic et al., 1999), cannot be used to determine the absolute amplitude of the dendritic electrical signal. The sensitivity of the dye $\Delta F / F$ (a fractional change in light intensity produced by a $100 \mathrm{mV}$ change in membrane potential) is an intrinsic physical characteristic of the membrane-bound fluorescent molecule and, as such, is thought to be the same in the cell body and in the dendrite. One is then tempted to conclude that by calibrating the optical signal in the cell body, with simultaneous whole-cell measurement, we could arrive at the exact amplitude of the electrical signal elsewhere on the dendritic tree. In fact, that would be possible if the voltagesensitive dye was bound only to the excitable plasma membrane, as in experiments using extracellular staining of neurons (Ross and Krauthamer, 1984; Meyer et al., 1997; Bullen and Saggau, 1999). Unfortunately, when applied intracellularly, voltage- sensitive dyes indiscriminately bind to all lipid bilayers, those in the plasma membrane, as well as those that make intracellular organelles. Fluorescent molecules bound to intracellular membranes do not experience changes in the electrical field during the neuronal electrical activity and therefore do not participate in the optical signal $(\Delta F)$. At the same time, these inert (nonreporting) dye molecules contribute significantly to the resting light level $(F)$ and therefore strongly affect the actual size of the optical signal expressed as $\Delta F / F$. As the amount of intracellular membrane varies among different cellular compartments (soma-dendrite), so does the fraction of the inert (nonreporting) dye (Zecevic and Antic, 1998). As a result, an AP $(\sim 100 \mathrm{mV})$ in the soma produces an optical signal of $<2 \%(\Delta F / F)$ in amplitude, whereas in the distal basal dendrite of the same neuron, the AP-associated optical signal is $\sim 4 \% \Delta F / F$ (Antic, 2003). The AP-associated optical signal in the dendrite is approximately two times larger than that in the soma, not because the amplitude of the dendritic AP is 200 $\mathrm{mV}$ but because the ratio of "reporting" and "nonreporting" dye molecules is at least two times better in dendrites than in the soma.

Although voltage-sensitive dyes cannot be used to determine the absolute size of the dendritic signal in millivolts, they can be used to obtain crude estimates of the relative difference in the depolarization amplitude between selected cellular compartments. Under the assumption that dendrites of the same type (such as basal dendrites), at the same distance form the dye injection point (cell body), have a similar ratio of the reporting and nonreporting dye, it is possible to compare the size of the voltagedye signal between look-alike (homogenous) dendritic compartments. In Figure $7 B$, rectangles 1 and 2 mark two homogeneous dendritic segments. Both segments belong to basal branches of the dendritic tree and are at approximately the same distance $(\sim 110 \mu \mathrm{m})$ from the center of the soma. In this and in all other experiments, in which two or more twin basal branches were in the visual field, the glutamate-evoked optical signal in the nontarget dendrite (Fig. $7 C$, ROI 2$)$ was only a fraction $(0.35 \pm 0.09$; $n=4$ neurons; $n=6$ pairs of dendrites) of the optical signal measured in the target branch (ROI 1). In all neurons treated with TTX, the optical signal obtained in the target dendrite (Fig. $7 E$, ROI 1) always preceded that in the soma (ROI 0), or in the nontarget dendrites (ROI 2), by several milliseconds $(8.3 \pm 3.7$ $\mathrm{ms} ; n=9)$. These data indicate that the source of depolarization was in the target dendrite, whereas the nontarget branches received cable-filtered depolarization signal from the cell body (BPP).

To determine whether the input-output function of the remote dendritic segment has been saturated, in nine TTX-treated neurons, the intensity of the glutamate pulse was increased beyond the threshold for the generation of the dendritic plateau. A gradual increase in the iontophoretic current (in the suprathreshold range) did not contribute significantly to the amplitude of the dendritic voltage signal, but it did invariably increase the dura- 
tion of the plateau phase in the dendrite as well as in the cell body (Fig. 7D). In three TTX-treated neurons, glutamate iontophoresis was applied on a basal dendrite starting at very small (subthreshold) intensities (Fig. 8). In subsequent sweeps, we monitored the transition of the evoked response from postsynaptic potential (sub.) to plateau potential (sup.), both in the cell body (Fig. $8 \mathrm{~B}$, ROI 0 ) and the target dendrite (ROI 1), simultaneously. Amplitudes of membrane potential transients in the cell body (expressed in millivolts, relative to resting $V_{\mathrm{m}}$ ) correlated well with those in the remote region of the basal dendrite (expressed as percentage of $\Delta F / F$ ) (Fig. $8 C)$. The correlation coefficients for three neurons in this data set were $0.977,0.980$, and 0.966 ( $n=7-8$ successive glutamate levels). Once the glutamate concentrations for plateau generation were reached (Fig. 8 , sup.), an additional increase in stimulus intensity did not contribute significantly to the amplitude of the dendritic (ROI 1) or somatic (ROI 0) membrane potential change. These findings suggest that the features of the neuronal input-output function, described in Figure 2 (the saturation of the somatic response followed by a linear increase in duration of the somatic plateau depolarization), are precisely derived from the dendritic input-output function.

\section{Discussion}

\section{The magnitude of the glutamatergic input in basal dendrites} Communication between neocortical pyramidal neurons is established almost exclusively through glutamatergic synapses that impinge on basal dendrites (Markram, 1997; Feldmeyer et al., 2002; Lubke et al., 2003). In addition to this massive cortical glutamatergic drive, basal dendrites are the primary target of glutamate-mediated synaptic inputs from extracortical sources (Herkenham, 1980; Agmon and Connors, 1991; Kuroda et al., 1998). It is thus important to examine how glutamatergic excitatory inputs are perceived by basal dendrites. In general, two technical limitations preclude our understanding of information processing in individual neurons. First, it is our inability to provide a well-defined synaptic input: a synaptic input that would be restricted to a particular dendritic spot of our choice, which will not alternate in amplitude because of presynaptic mechanisms of depression or facilitation, and the gain of which would be under the fine control of the experimenter. A second technical obstacle is our inability to routinely record membrane potential transients from thin dendritic branches. Ideally, one would like to be able to measure electrical signals simultaneously from multiple sites, throughout the dendritic tree, during the course of synaptic integration (Djurisic et al., 2003). In our laboratory, we approach these two technical problems by using (1) glutamate iontophoresis from sharp glass pipettes and (2) internally applied voltagesensitive dyes. Sharp electrode glutamate iontophoresis allows precise positioning, great reliability (Fig. $4 \mathrm{~B}$ ), and fine control of the amount of injected glutamate (Figs. 2, 8). Voltage-sensitive dyes allow fast multisite measurements of membrane potential changes along one or several basal branches at the same time (Fig.
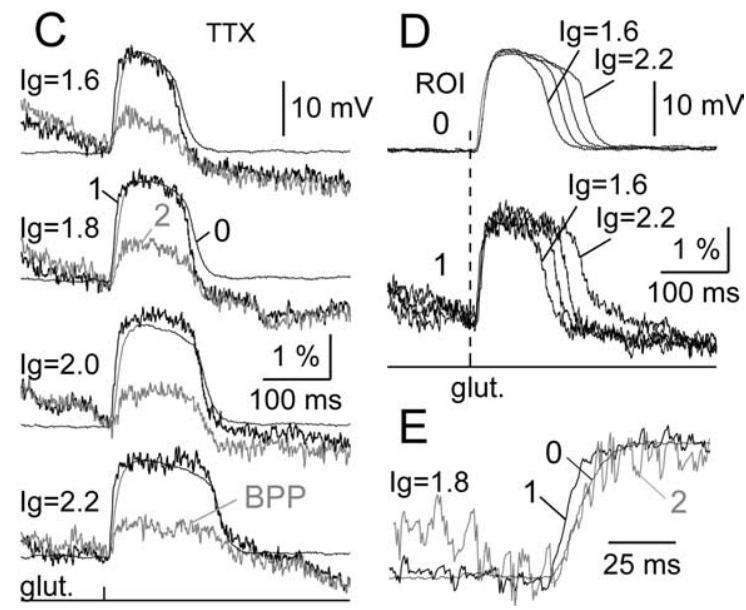$$
\text { glut. }
$$

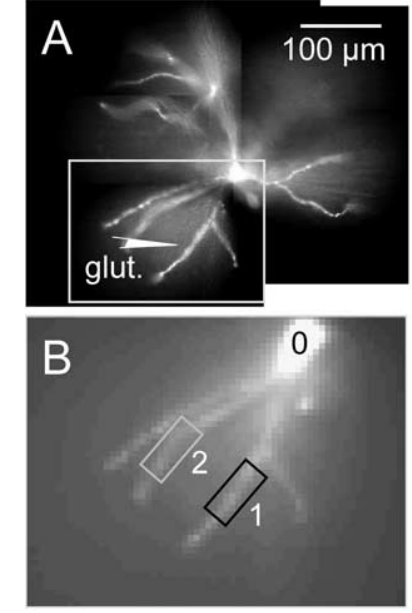

Figure 7. Saturation of the dendritic membrane potential. $\boldsymbol{A}$, Composite microphotograph of a layer V pyramidal cell stained with JPW1114. The schematic drawing indicates the position of the glutamate-filled pipette $(110 \mu \mathrm{m}$ from the center of the where optical signals were selected for spatial averaging and display. Each box encompasses 16 pixels. C, The glutamate iontophoretic current was increased from 1.6 to $2.2 \mu \mathrm{A}$ in increments of 0.2 , whereas membrane (

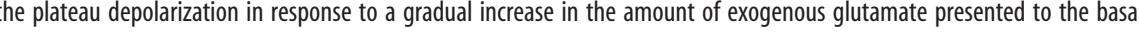
dendis at $50 \%$ plateau amplitude). glut., Glutamate.

6). These two experimental methods were used to explore the sequence of events in the basal dendritic tree during sustained plateau depolarizations (in vitro-evoked UP state-like depolarizations). Our experiments revealed that the slow component of the somatic signal is in strict correlation with the dendritic membrane potential transient in terms of the (1) amplitude (Fig. 8), (2) timing (Fig. 7), and (3) duration of the glutamate-evoked event (Fig. 5).

\section{Plateau potentials and dendritic input-output function}

Plateau-like all-or-none responses were observed previously after brief glutamatergic stimulations of the basal dendrites of neocortical pyramidal neurons (Schiller et al., 2000) and the apical tuft dendrites of hippocampal pyramidal neurons (Wei et al., 2001). However, in these studies, an increase in the duration of the glutamate-evoked plateau was not studied systematically. In the present study, the plateaus in the cell body (Fig. 2), as well as in the remote region of the basal dendrite (Fig. 7), grew longer in a linear manner, in response to a linear increase in the glutamate iontophoretic current. Therefore, it seems that basal dendrites of prefrontal pyramidal neurons use two different input-output functions when processing very strong synaptic inputs: (1) basal dendrites operate in a binary regimen ( 0 or 1 , DOWN or UP) in regard to the amplitude of the glutamate-evoked electrical signal (Fig. 2E); and (2) basal dendrites operate in a linear regimen with respect to the duration of the glutamate-evoked electrical transient (Fig. $2 F$ ).

Dendritic binary behavior has been first introduced and studied in computer simulations (Mel, 1993; Softky and Koch, 1993; Segev and Rall, 1998; Rhodes, 1999). Dendritic nonlinearity has been implicated to underlie orientation and disparity tuning in pyramidal neurons of the visual cortex (Archie and Mel, 2000), as well as learning and long-term memory storage (Poirazi and Mel, 2001). Of a particular interest for the present study are biophys- 

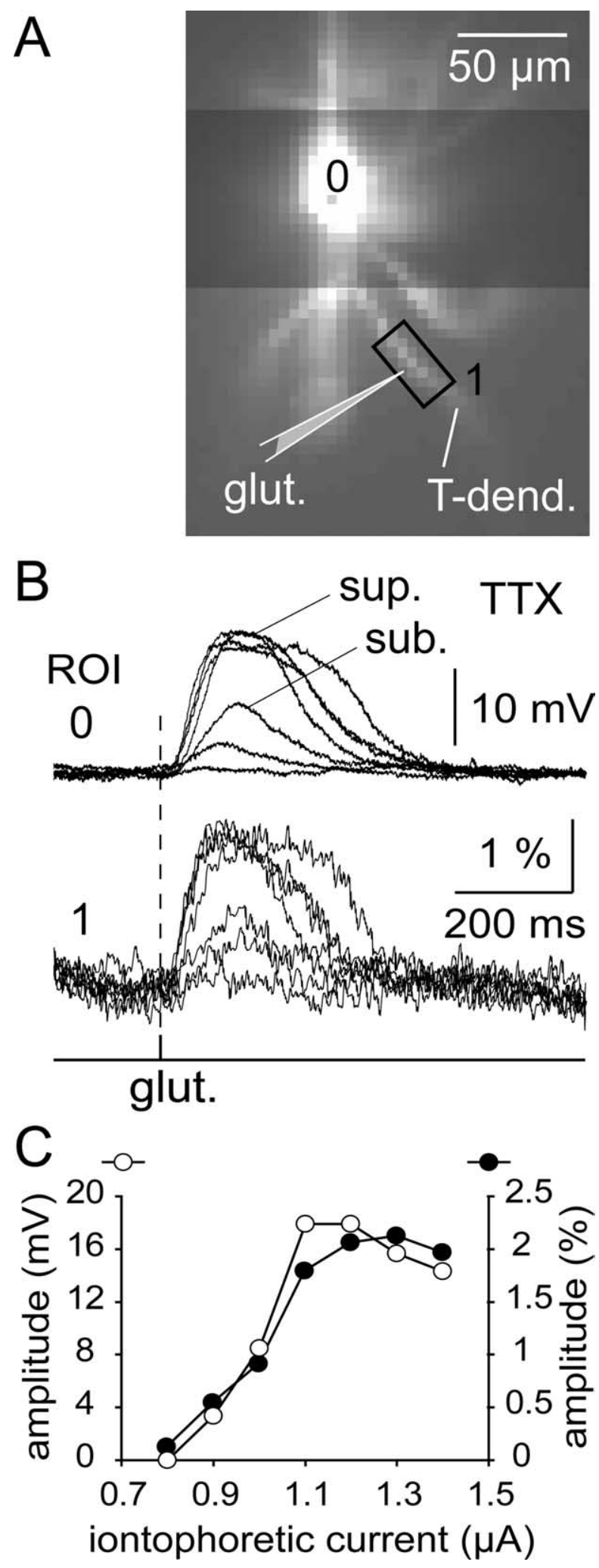

Figure 8. Correlation between dendritic and somatic depolarization amplitudes. $\boldsymbol{A}$, Composite fluorescent image of a layer $V$ pyramidal cell $(80 \times 80$ pixels). The schematic drawing indicates the position of the glutamate-filled pipette ( $90 \mu \mathrm{m}$ from the center of the soma). $\boldsymbol{B}$, Whole-cell recordings from the cell body (ROI 0 ) are aligned with voltage-sensitive dye recordings obtained from the glutamate stimulation site (ROI 1). Sixteen pixels are spatially averaged in the area marked with the rectangle in $\boldsymbol{A}$. The vertical dashed line marks the timing of the glutamate pulse (duration, $5 \mathrm{~ms}$; intensity varied from 0.8 to $1.4 \mu \mathrm{A}$ ). $\Pi \mathrm{TX}, 1 \mu \mathrm{M}$. $\mathrm{C}$, The plot of signal amplitudes measured in the cell body (in millivolts; white circles) and in the dendrite [in percentage ( $\Delta F / F)$; black circles]. glut., Glutamate; T-den., target dendrite. ical models of persistent neuronal activity that are based on recurrent excitation (feedback). The network models of persistent neuronal activity normally relay on precisely tuned feedback (Seung et al., 2000). A change in synaptic weight, as small as $1-2 \%$, can completely disrupt the stability of the model, and neuronal activity either goes into saturation or decays to zero. The sensitivity problem was alleviated, and overall performance of the model was improved when dendrites had been given bistable properties (Goldman et al., 2003), which suggests that dendritic binary nature, as a general mechanism, might play an important role in the generation and maintenance of persistent neuronal activity in different parts of the CNS including the striatum (Wilson, 1993; Vergara et al., 2003) and prefrontal cortex (Milojkovic et al., 2004).

Recent voltage-clamp analysis of glutamate-evoked currents in basal dendrites revealed their regenerative nature (Schiller et al., 2000). Our experiments (Fig. 3) brought four important novelties. First, we have chosen to study glutamate-evoked transmembrane currents that had been confirmed to trigger the neuronal UP state-like depolarization in current-clamp mode (Fig. $3 A$, sweep 0 ). The importance of these currents for the neuronal output was therefore clearly defined in our experiments. Second, the recordings revealed fast initial spikelets (Fig. 3A, traces 1-3) that were previously thought to be an exclusive feature of CA1 pyramidal cell basal dendrites (Ariav et al., 2003). Third, our results show that the glutamate-evoked current has two distinguishable components: (1) a fast square-shaped plateau (spike) that is riding on top of (2) a smaller and slower voltageinsensitive foot (Fig. 3B, -82). The transition from subthreshold to threshold response in the dendritic input-output function obtained in current clamp (Fig. 2D) seems to correspond well with the transition between "no spike" and spike response in voltage-clamp experiments (Fig. $3 A$ ). Finally, despite being a clearly all-or-none phenomenon in terms of amplitude (Fig. $3 C, D)$, the duration of glutamate-evoked spikes shows great sensitivity to voltage perturbations (Fig. 3E). The duration of cortical UP states in vivo has been found previously to correlate with the resting membrane potential (Cowan and Wilson, 1994).

\section{Plateau potentials and UP states}

There is plenty of evidence that neuronal persistent activity (UP state) is a network phenomenon (Amzica and Steriade, 1995; Timofeev et al., 2000) and that myriads of inhibitory inputs coincide with patterned excitatory waves during the cortical UP state (Steriade et al., 1993a; Contreras et al., 1997a; Sanchez-Vives and McCormick, 2000; Seamans et al., 2003). The abundance of inhibitory inputs during the UP phase is hardly surprising given the fact that $\sim 88 \%$ of recorded neurons in vivo (Steriade et al., $1993 \mathrm{a})$, and nearly all $(\sim 100 \%)$ of the neuronal elements in an in vitro model of the slow-wave sleep (Sanchez-Vives and McCormick, 2000), are synchronously activated during the cortical UP state. It has been well established that activation of almost any given cortical afferent pathway produces excitation followed by inhibition in pyramidal neurons (Kita and Oomura, 1981; McCormick, 1989; Zhu and Connors, 1999). To test whether inhibitory synaptic inputs are a fundamental cellular mechanism for maintaining the stable sustained depolarized state, we blocked APs with TTX and stimulated basal dendrites with exogenous glutamate. In the absence of any network-generated feedback inhibition, pyramidal neurons remained in a stable depolarized state for several hundred milliseconds (Fig. 4C). Inhibitory interneurons are critical for (1) shaping the spread of excitation over cortical areas (Telfeian and Connors, 1998; Gibson et al., 1999), 
(2) a precise temporal control of individual APs (Timofeev et al., 2001), and (3) generation of network rhythms (Jefferys et al., 1996; McBain and Fisahn, 2001). The question remains whether IPSPs are well suited for maintaining the stable sustained somatic depolarization during the UP state (Shu et al., 2003). As pointed out by Stern et al. (1997), "inhibition is not a robust mechanism for maintaining the somatic membrane potential at one constant level, because to do so it must be very precisely adjusted to match the level of excitation." We ought to be asking whether IPSPs perhaps work in "cooperation" with other membrane mechanisms to perform this delicate task (McCormick et al., 2003). The list of membrane mechanisms auxiliary to feedback inhibition may include intrinsic $\mathrm{K}^{+}$currents (Wilson and Kawaguchi, 1996; Contreras et al., 1997b; Cai et al., 2004) but also the saturation of the dendritic excitation (Figs. $7 D, 8 B$ ).

The transition of cortical pyramidal cells from UP to DOWN state was thought to result in response to the strong activation of inhibitory neurons (Brunel and Wang, 2001; Shu et al., 2003), or perhaps in response to synchronized activation of the interconnected neurons, thereby leading to a synchronized refractory period (Gutkin et al., 2001; Shu et al., 2003). In our experiments, pyramidal neurons swiftly returned from UP state-like depolarization in the absence of inhibitory inputs or other AP-dependent membrane mechanisms (Fig. $4 B$, TTX). The finding that the breakdown of the somatic depolarized state occurred in strict correlation with the collapse of the dendritic plateau potential (Figs. 5-8) supports an alternative view for the transition from UP to DOWN state. This transition may be a direct consequence of the breakdown of dendritic regenerative plateau potential. In this scenario, the cell body shifts from DOWN to UP state after the generation of the dendritic plateau potential. It stays in the UP state as long as the dendritic plateau lasts, and it collapses with the collapse of the dendritic plateau potential. In this way, the slow component of the somatic signal during UP states is just a reflection of a flamboyant integration process occurring somewhere in the dendritic tree.

\section{References}

Agmon A, Connors BW (1991) Thalamocortical responses of mouse somatosensory (barrel) cortex in vitro. Neuroscience 41:365-379.

Amzica F, Steriade M (1995) Disconnection of intracortical synaptic linkages disrupts synchronization of a slow oscillation. J Neurosci 15:4658-4677.

Antic S, Major G, Zecevic D (1999) Fast optical recordings of membrane potential changes from dendrites of pyramidal neurons. J Neurophysiol 82:1615-1621.

Antic SD (2003) Action potentials in basal and oblique dendrites of rat neocortical pyramidal neurons. J Physiol (Lond) 550:35-50.

Archie KA, Mel BW (2000) A model for intradendritic computation of binocular disparity. Nat Neurosci 3:54-63.

Ariav G, Polsky A, Schiller J (2003) Submillisecond precision of the inputoutput transformation function mediated by fast sodium dendritic spikes in basal dendrites of CA1 pyramidal neurons. J Neurosci 23:7750-7758.

Brunel N, Wang XJ (2001) Effects of neuromodulation in a cortical network model of object working memory dominated by recurrent inhibition. J Comput Neurosci 11:63-85.

Bullen A, Saggau P (1999) High-speed, random-access fluorescence microscopy: II. Fast quantitative measurements with voltage-sensitive dyes. Biophys J 76:2272-2287.

Cai X, Liang CW, Muralidharan S, Kao JP, Tang CM, Thompson SM (2004) Unique roles of SK and Kv4.2 potassium channels in dendritic integration. Neuron 44:351-364.

Compte A, Sanchez-Vives MV, McCormick DA, Wang XJ (2003) Cellular and network mechanisms of slow oscillatory activity $(<1 \mathrm{~Hz})$ and wave propagations in a cortical network model. J Neurophysiol 89:2707-2725.

Contreras D, Destexhe A, Steriade M (1997a) Intracellular and computational characterization of the intracortical inhibitory control of synchronized thalamic inputs in vivo. J Neurophysiol 78:335-350.
Contreras D, Durmuller N, Steriade M (1997b) Plateau potentials in cat neocortical association cells in vivo: synaptic control of dendritic excitability. Eur J Neurosci 9:2588-2595.

Cowan RL, Wilson CJ (1994) Spontaneous firing patterns and axonal projections of single corticostriatal neurons in the rat medial agranular cortex. J Neurophysiol 71:17-32.

Danbolt NC (2001) Glutamate uptake. Prog Neurobiol 65:1-105.

Djurisic M, Zochowski M, Wachowiak M, Falk CX, Cohen LB, Zecevic D (2003) Optical monitoring of neural activity using voltage-sensitive dyes. Methods Enzymol 361:423-451.

Feldmeyer D, Lubke J, Silver RA, Sakmann B (2002) Synaptic connections between layer 4 spiny neurone-layer $2 / 3$ pyramidal cell pairs in juvenile rat barrel cortex: physiology and anatomy of interlaminar signalling within a cortical column. J Physiol (Lond) 538:803-822.

Franks KM, Bartol Jr TM, Sejnowski TJ (2002) A Monte Carlo model reveals independent signaling at central glutamatergic synapses. Biophys J 83:2333-2348.

Gibson JR, Beierlein M, Connors BW (1999) Two networks of electrically coupled inhibitory neurons in neocortex. Nature 402:75-79.

Goldman MS, Levine JH, Major G, Tank DW, Seung HS (2003) Robust persistent neural activity in a model integrator with multiple hysteretic dendrites per neuron. Cereb Cortex 13:1185-1195.

Gutkin BS, Laing CR, Colby CL, Chow CC, Ermentrout GB (2001) Turning on and off with excitation: the role of spike-timing asynchrony and synchrony in sustained neural activity. J Comput Neurosci 11:121-134.

Herkenham M (1980) Laminar organization of thalamic projections to the rat neocortex. Science 207:532-535.

Jefferys JG, Traub RD, Whittington MA (1996) Neuronal networks for induced " $40 \mathrm{~Hz}$ " rhythms. Trends Neurosci 19:202-208.

Kita H, Oomura Y (1981) Reciprocal connections between the lateral hypothalamus and the frontal complex in the rat: electrophysiological and anatomical observations. Brain Res 213:1-16.

Kuroda M, Yokofujita J, Murakami K (1998) An ultrastructural study of the neural circuit between the prefrontal cortex and the mediodorsal nucleus of the thalamus. Prog Neurobiol 54:417-458.

Larkum ME, Kaiser KM, Sakmann B (1999) Calcium electrogenesis in distal apical dendrites of layer 5 pyramidal cells at a critical frequency of backpropagating action potentials. Proc Natl Acad Sci USA 96:14600-14604.

Larkum ME, Zhu JJ, Sakmann B (2001) Dendritic mechanisms underlying the coupling of the dendritic with the axonal action potential initiation zone of adult rat layer 5 pyramidal neurons. J Physiol (Lond) 533:447-466.

Lewis BL, O’Donnell P (2000) Ventral tegmental area afferents to the prefrontal cortex maintain membrane potential "up" states in pyramidal neurons via D(1) dopamine receptors. Cereb Cortex 10:1168-1175.

Lubke J, Roth A, Feldmeyer D, Sakmann B (2003) Morphometric analysis of the columnar innervation domain of neurons connecting layer 4 and layer 2/3 of juvenile rat barrel cortex. Cereb Cortex 13:1051-1063.

Markram H (1997) A network of tufted layer 5 pyramidal neurons. Cereb Cortex 7:523-533.

Matthews CC, Zielke HR, Wollack JB, Fishman PS (2000) Enzymatic degradation protects neurons from glutamate excitotoxicity. J Neurochem 75:1045-1052.

McBain CJ, Fisahn A (2001) Interneurons unbound. Nat Rev Neurosci 2:11-23.

McCormick DA (1989) GABA as an inhibitory neurotransmitter in human cerebral cortex. J Neurophysiol 62:1018-1027.

McCormick DA, Shu YS, Hasenstaub A, Sanchez-Vives M, Badoual M, Bal T (2003) Persistent cortical activity: mechanisms of generation and effects on neuronal excitability. Cereb Cortex 13:1219-1231.

Mel BW (1993) Synaptic integration in an excitable dendritic tree. J Neurophysiol 70:1086-1101.

Metherate R, Ashe JH (1993) Ionic flux contributions to neocortical slow waves and nucleus basalis-mediated activation: whole-cell recordings in vivo. J Neurosci 13:5312-5323.

Meyer E, Muller CO, Fromherz P (1997) Cable properties of dendrites in hippocampal neurons of the rat mapped by a voltage-sensitive dye. Eur J Neurosci 9:778-785.

Milojkovic BA, Radojicic MS, Goldman-Rakic PS, Antic SD (2004) Burst generation in rat pyramidal neurones by regenerative potentials elicited in a restricted part of the basilar dendritic tree. J Physiol (Lond) 558:193-211.

Oakley JC, Schwindt PC, Crill WE (2001) Dendritic calcium spikes in layer 
5 pyramidal neurons amplify and limit transmission of ligand-gated dendritic current to soma. J Neurophysiol 86:514-527.

Poirazi P, Mel BW (2001) Impact of active dendrites and structural plasticity on the memory capacity of neural tissue. Neuron 29:779-796.

Rhodes PA (1999) Functional implications of active currents in the dendrites of pyramidal neuros. In: Cerebral cortex, Vol 3 (Ulinski PS, Jones EG, Peters A, eds), pp 139-200. New York: Kluwer Academic.

Ross WN, Krauthamer V (1984) Optical measurements of potential changes in axons and processes of neurons of a barnacle ganglion. J Neurosci 4:659-672.

Sanchez-Vives MV, McCormick DA (2000) Cellular and network mechanisms of rhythmic recurrent activity in neocortex. Nat Neurosci 3:1027-1034.

Schiller J, Helmchen F, Sakmann B (1995) Spatial profile of dendritic calcium transients evoked by action potentials in rat neocortical pyramidal neurones. J Physiol 487:583-600.

Schiller J, Major G, Koester HJ, Schiller Y (2000) NMDA spikes in basal dendrites of cortical pyramidal neurons. Nature 404:285-289.

Schwindt P, Crill W (1999) Mechanisms underlying burst and regular spiking evoked by dendritic depolarization in layer 5 cortical pyramidal neurons. J Neurophysiol 81:1341-1354.

Seamans JK, Nogueira L, Lavin A (2003) Synaptic basis of persistent activity in prefrontal cortex in vivo and in organotypic cultures. Cereb Cortex 13:1242-1250.

Segev I, Rall W (1998) Excitable dendrites and spines: earlier theoretical insights elucidate recent direct observations. Trends Neurosci 21:453-460.

Seung HS, Lee DD, Reis BY, Tank DW (2000) Stability of the memory of eye position in a recurrent network of conductance-based model neurons. Neuron 26:259-271.

Shu YS, Hasenstaub A, McCormick DA (2003) Turning on and off recurrent balanced cortical activity. Nature 423:288-293.

Softky WR, Koch C (1993) The highly irregular firing of cortical cells is inconsistent with temporal integration of random EPSPs. J Neurosci 13:334-350.

Steriade M, Nunez A, Amzica F (1993a) A novel slow ( $<1 \mathrm{~Hz}$ ) oscillation of neocortical neurons in vivo: depolarizing and hyperpolarizing components. J Neurosci 13:3252-3265.

Steriade M, Nunez A, Amzica F (1993b) Intracellular analysis of relations between the slow $(<1 \mathrm{~Hz})$ neocortical oscillation and other sleep rhythms of the electroencephalogram. J Neurosci 13:3266-3283.
Stern EA, Kincaid AE, Wilson CJ (1997) Spontaneous subthreshold membrane potential fluctuations and action potential variability of rat corticostriatal and striatal neurons in vivo. J Neurophysiol 77:1697-1715.

Stuart G, Sakmann B (1995) Amplification of EPSPs by axosomatic sodium channels in neocortical pyramidal neurons. Neuron 15:1065-1076.

Telfeian AE, Connors BW (1998) Layer-specific pathways for the horizontal propagation of epileptiform discharges in neocortex. Epilepsia 39:700-708.

Timofeev I, Grenier F, Bazhenov M, Sejnowski TJ, Steriade M (2000) Origin of slow cortical oscillations in deafferented cortical slabs. Cereb Cortex 10:1185-1199.

Timofeev I, Grenier F, Steriade M (2001) Disfacilitation and active inhibition in the neocortex during the natural sleep-wake cycle: an intracellular study. Proc Natl Acad Sci USA 98:1924-1929.

Vergara R, Rick C, Hernandez-Lopez S, Laville JA, Guzman JN, Galarraga E, Surmeier DJ, Bargas J (2003) Spontaneous voltage oscillations in striatal projection neurons in a rat corticostriatal slice. J Physiol (Lond) 553:169-182.

Wei DS, Mei YA, Bagal A, Kao JP, Thompson SM, Tang CM (2001) Compartmentalized and binary behavior of terminal dendrites in hippocampal pyramidal neurons. Science 293:2272-2275.

West AR, Moore H, Grace AA (2002) Direct examination of local regulation of membrane activity in striatal and prefrontal cortical neurons in vivo using simultaneous intracellular recording and microdialysis. J Pharmacol Exp Ther 301:867-877.

Wilson CJ (1993) The generation of natural firing patterns in neostriatal neurons. In: Progress in brain research (Arbuthnott GW, Emson PC, eds), pp 277-297. Amsterdam: Elsevier.

Wilson CJ, Kawaguchi Y (1996) The origins of two-state spontaneous membrane potential fluctuations of neostriatal spiny neurons. J Neurosci 16:2397-2410.

Yang CR, Seamans JK, Gorelova N (1996) Electrophysiological and morphological properties of layers V-VI principal pyramidal cells in rat prefrontal cortex in vitro. J Neurosci 16:1904-1921.

Zecevic D, Antic S (1998) Fast optical measurement of membrane potential changes at multiple sites on an individual nerve cell. Histochemical J 30:197-216.

Zhu JJ, Connors BW (1999) Intrinsic firing patterns and whisker-evoked synaptic responses of neurons in the rat barrel cortex. J Neurophysiol 81:1171-1183. 